\title{
Intelligent agent based framework to augment warehouse management systems for dynamic demand environments
}

\author{
Tania Binos \\ RMIT University \\ Melbourne, Australia \\ binos.tania@rmit.edu.au
}

\section{Vince Bruno}

RMIT University

Melbourne, Australia

\author{
Arthur Adamopoulos \\ RMIT University \\ Melbourne, Australia
}

\begin{abstract}
Warehouses are being impacted by increasing e-commerce and omni-channel commerce. The design of current WMSs (Warehouse Management Systems) may not be suitable to this mode of operation. The golden rule of material handling is smooth product flow, but there are dayto-day operational issues that occur in the warehouse that can impact this and order fulfilment, resulting in disruptions. Standard operational process is paramount to warehouse operational control but may preclude a dynamic response to real-time operational constraints. The growth of IoT (Internet of Things) sensor and data analytics technology provide new opportunities for designing warehouse management systems that detect and reorganise around real-time constraints to mitigate the impact of day-to-day warehouse operational issues. This paper presents the design and development stage of a design science methodology of an intelligent agent framework for basic warehouse management systems. This framework is distributed, is structured around operational constraints and includes the human operator at operational and decision support levels. An agent based simulation was built to demonstrate the viability of the framework.
\end{abstract}

Keywords warehouse management systems, distributed intelligence, software agents, decision support.

\section{Introduction}

Increasingly, the future of warehouses is considered to be completely automated and humanless. Not all warehouses can feasibly be automated, however. The expense of automation can preclude this solution in many smaller warehouses. Products that are odd shapes and bulky can also be difficult to handle robotically. This study proposes that advances in IoT and AI can bridge the gap between existing largely manual warehouses and full automation.

Changes in product make up and e-commerce orders present new issues for warehouses and distribution centres. The impact of expanding e-commerce and omni-channel business models (Michel, 2016) create a new way of working that is characterised by increasingly changing customer demands, higher product variety, smaller order size, the expectation of reliably shorter response times (Lu, Giannikas, McFarlane, \& Hyde, 2014) and irregular order arrival (Leung et al., 2018). Storing a wider variety of products in a warehouse in smaller quantities 
can mean that the way the warehouse and its systems work is different (Davarzani \& Norrman, 2015). In fact, only $10 \%$ of distribution centres exclusively handle pallets now, while $66 \%$ handle a mixture of pallets, cases, split cases, and pieces (Michel, 2018a). Many warehouse management systems (WMS) are not designed for small orders picked in piece units. In the 2018 Warehouse/Distribution Center (DC) Equipment Survey(Michel, 2018b), 29\% of respondents indicated that the most common process for e-commerce fulfilment in their operation was "buy online and ship to customer from DC" although there was growth in "buy online and ship to customer from vendor". This suggests that the DC might be struggling with the processing of e-commerce orders. This pressure may see the default of members of the supply chain and this can alter the behaviour and the exposure to risk of other members of the supply chain (Gibilaro \& Mattarocci, 2019).

Warehouse design including operational processes are predominantly based on current and projected future demand (De Koster, Le-Duc, \& Roodbergen, 2007) and changes to design after construction are costly (Gu, Goetschalckx, \& McGinnis, 2007). The warehouse control systems and software that operate them are costly to implement and change and require considerable training and support (Min, 2006). Warehouses are designed to be well-defined process-driven product flow machines (Bartholdi \& Hackman, 2008). Their aim is to achieve maximum throughput with minimum investment and operational costs (Bartholdi \& Hackman, 2008; Rouwenhorst et al., 2000; van den Berg \& Zijm, 1999). However, their inability to flexibly respond to dynamic changes in demand leads to common issues such as aisle congestion, mistimed replenishments of the pick face, short-picks and double handling (Gong \& De Koster 2011, Bartholdi \& Hackman 2008; Gu, Goetschalckx \& McGinnis 2009). Dynamic responses are needed when the fixed constraints of the environment have been impacted.

In dynamic environments, a small number of constraints can lead to operational issues that have a large impact on the warehouse throughput as a whole. These can impact warehouse performance if not detected and corrected in a timely manner. These include:

- The operator is unable to complete order selection or replenishment of a selection location. Potential causes:

- $\quad$ Congestion (for selector or forklift operator) (M. Zhang, Batta, \& Nagi, 2009)

- Inventory system inaccuracies (inventory record does not match reality). Many factors can cause inventory inaccuracies including visual complexity (Barratt, Kull, \& Sodero, 2018).

- Inaccurate product and packaging dimension data that can misrepresent the actual height and weight of a storage unit. SKU weights and dimensions can be hard to maintain due to supplier changes(Michel, 2016)

- Slow replenishments

- A replenishment that is triggered too early or too late has flow-on effects to picking (Richards, 2014; Rushton, Croucher, \& Baker, 2014) such as overfull location (too early) or short-pick (too late). Short picks require extra order selection trips and delay order completion.

- $\quad$ Slow receiving/checking processes.

- A failure to prioritise stock that will be required sooner based on order demand (Richards, 2014) 
- $\quad$ Picking from incorrect location, Incorrect stock in location, Over-picking, Underpicking

- Identification of this issue (occurring even with bar-coding, RF (Radio Frequency) scanning and voice picking technology) can highlight incorrect case or inner case barcoding or operator error. Picking inaccuracies that are not discovered prior to shipping can result in returns, repeat deliveries and loss of goodwill (García, Chang, Abarca, \& Oh, 2007). Pick error rates were reported at between $0.02 \%$ and $0.05 \%$ by companies participating in a qualitative analysis of work schedule deviances (Glock, Grosse, Elbert, \& Franzke, 2017).

- $\quad$ Staging dock congestion

- $\quad$ Badly managed staging areas can lead to congestion and scattered staging of picked orders causing issues when loading trucks for delivery. Picked goods for dispatch should not be ready too early as this causes congestion and should also not be late as this holds up the loading process and the despatch door (Walker, 2018)

As with most errors that occur with flow-on effects in a system, prevention or detection and correction in a real-time or near real-time manner is desirable. This is especially important in the order fulfilment space, where truck scheduling and delivery time windows need to be met and returns are costly or become losses (especially with high value stock).

Current warehouse management systems (WMS) are generally top-down, centralised systems and their decision support functions are the same. The disadvantages of central control include a single point of failure, inconsistent speed of response and a high dependency in the structure (Haneyah, Schutten, Schuur, \& Zijm, 2013). Most of these software packages deal with producing tasks to completely pick and despatch a batch of customer orders in a given time period. Inventory management and interfaces to transport and billing (ERP) systems are managed as part of order fulfilment. The dynamic operational control is mainly done by humans via summary reports and screens that require human intervention and that are highly customised (Haneyah et al., 2013). This study sought to find a better way to design warehouse management systems, utilizing intelligent agents. It sought to answer the following research question:

How can distributed intelligent agents augment warehouse operations to mitigate the impact of dynamic day-to-day issues?

The purpose of this paper is to propose a distributed model for designing warehouse management systems that detects and reorganises around real-time constraints to mitigate the impact of day to day warehouse operational issues. The model is depicted in Figure 1. 


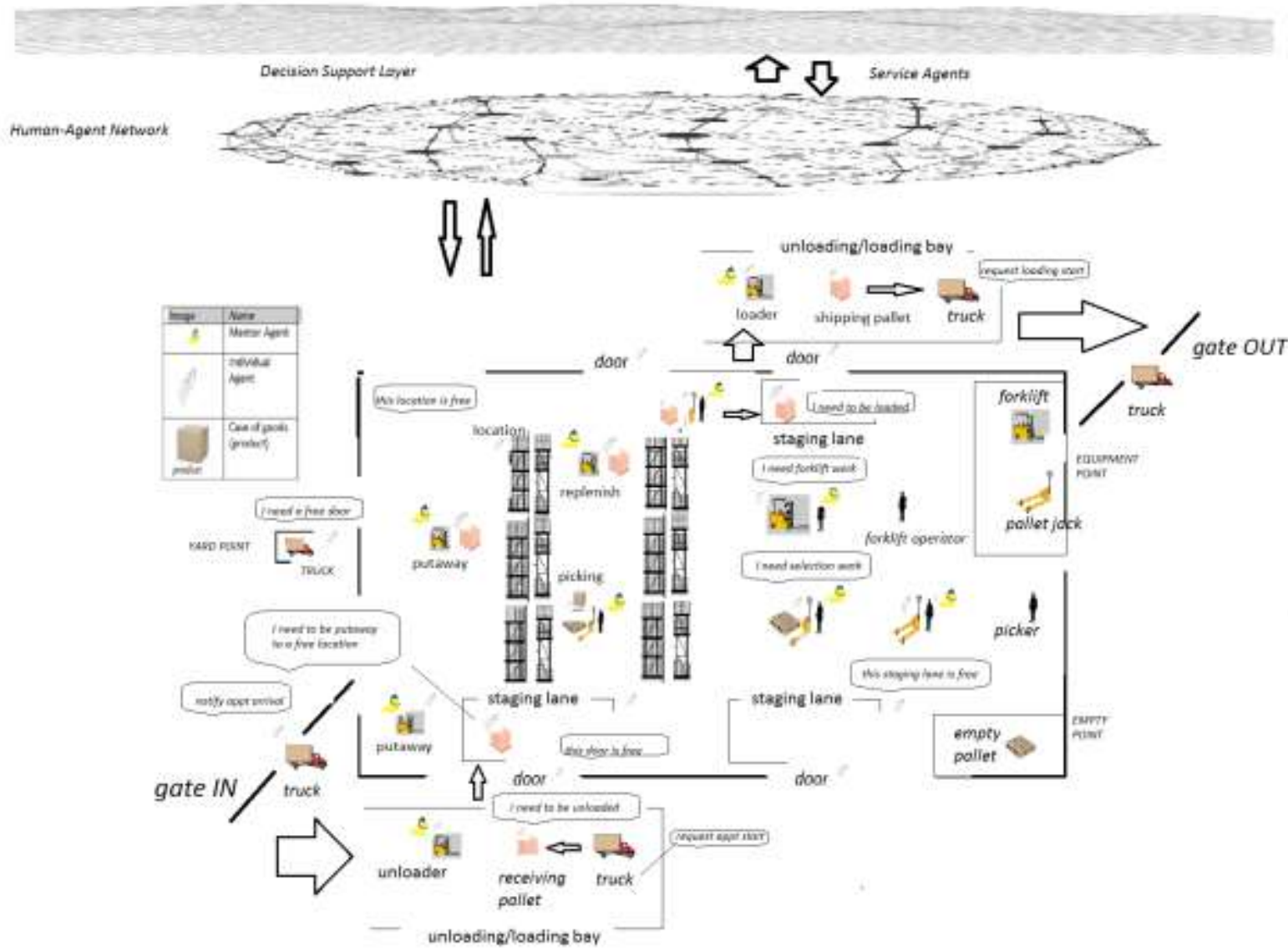

Figure 1 Conceptual Model of proposed WMS design framework

The elements of this model include:

- $\quad$ Distributed intelligence via cooperative agents

- $\quad$ Real time feedback/detection mechanisms

- Incorporate real-time constraints in the framework

- Embed the human operator as an actor in the framework with consideration of:

- $\quad$ Physical, mental and social real-time constraints

- $\quad$ The information required at different levels for decision support

- $\quad$ Adaptive learning based on trends for decision support

- $\quad$ Allow for AI algorithms to be plugged in where applicable and appropriate based on the temporal environment.

\section{Literature Review}

The challenges faced in decision support for complex systems such as warehouses are: infeasibility (all the information is not available in one place), impracticality (it would not be practical to come up with a centrally determined optimal solution), inadvisability (even if you had such a solution it would not be advisable) (Marik \& McFarlane, 2005). Distributed intelligence (DI) as a bottom-up approach to solving these issues has been proposed to enhance flexibility and agility (García et al., 2007). DI is a system of elements that have a degree of autonomy of operation, can reason solely or jointly and can interpret the state of the 
environment and the intentions of other elements (McFarlane, Parlikad, Neely, \& Thorne, 2012). Distributed intelligence can be implemented using an intelligent agent framework (Shukla \& Frank Chen, 1996).

Decision making within distributed intelligence can be determined by the data visibility at each point or node of a system. Technologies such as RFID, Internet of Things (IoT), GPS and other tracking systems are making this easier (Ding, 2013; Karagiannaki, Papakiriakopoulos, \& Bardaki, 2011; Liu et al., 2013).

One can imagine a warehouse environment in which information from sensors is used to form a picture of the environment and make a decision in real-time. Estanjini, Lin, Li, Guo, and Paschalidis (2011) developed such a system to determine the next task to be allocated to forklift drivers in a grocery warehouse. The system used a sensor network, an information collection system, a localisation algorithm and dynamic programming to determine the next task to allocate to an available forklift. A dynamic programming technique - actor-critic algorithms was used to determine the optimal average cost in simulation and this value was used in the implementation in a case study. Dynamic programming is used in multi-dimensional problem spaces such as this one where finding an exact optimum solution is almost impossible.

One approach using product intelligence (Giannikas, Lu, McFarlane, \& Hyde, 2013) uses a product and a shelf agent to select an appropriate storage location. This introduces the concept of non-human agency in the warehouse giving agency to products and shelves and is an important aspect of actor-network theory (Latour, 2005). This "intelligent product" concept has been extended into concepts such as "communicating objects"(Trab et al., 2017) and "smart warehouses" (Ding, 2013).

A new model using multi-agent systems (MAS) and IoT is proposed by Reaidy, Gunasekaran, and Spalanzani (2015), responding to the growing norm of uncertainty in supply chains. This model uses a bottom-up approach in which simple functions with local objectives are distributed. Ambient intelligence is achieved through sensors, control science (expert systems) and telecommunications. Agent communication strategies may be cooperation, competition, coo-petition or comp-eration (Reaidy et al., 2015).

Trab et al. (2017) use the "communicating object" concept and IoT to deal with the safe handling of hazardous products in a warehouse. All actors in the warehouse are modelled including human operators. The proposed hybrid intelligent system (central and distributed elements) has three levels of interaction $\mathrm{O} 2 \mathrm{O}$ (object to object), $\mathrm{O} 2 \mathrm{H}$ (object to human) and $\mathrm{O} 2 \mathrm{E}$ (object to environment). Liu et al. (2013)) discusses full electronic and GPS tracking of all goods coming in and out of the warehouse using RFID, GPRS (GPS) and Zigbee WSN for tracking within the warehouse. A model for the safe handling of hazardous goods in warehouses is also proposed using RFID and IoT to track materials and notify of incorrect placement and to support storage decisions (L. Zhang, Alharbe, \& Atkins, 2016). An artificial neural network (ANN) is used for environment detection. What emerges from the above research is a model where information about the environment is pieced together from sensors and made sense of through algorithms and AI.

Multi-agent systems (MAS) have been studied in warehouse management systems (García et al., 2007; Kim, Graves, \& Heragu, 2002; Rubrico, Ota, Higashi, \& Tamura, 2006). A MAS system was proposed to control stock levels using information from inputs and outputs of a bedding company warehouse captured via RFID (García et al., 2007). The stock level control was done 
at the ERP level while the enhanced RFID/IMS system was used to improve warehouse inventory management and picking accuracy. This was a bottom up approach feeding decision support systems. Multi-agent systems that communicate have been used to optimise the assignment of a pick route to an available picker based on proximity to the first location to be picked (Gharbi, Zgaya, \& Hammadi, 2013). The location of the pickers is determined via RFID.

In one agent-based model presented for the movement of products from the main warehouse to storage in the production warehouse for use on the production line four agents are used: production, storage, forklift and main warehouse (Maka, Cupek, \& Wierzchanowski, 2011). The products are moved between the storage and production warehouses by forklift. As well as communication, there is some complex logic in scheduling which products should be moved, in what priority and in what sequence they should be loaded and unloaded from the forklift. The authors make an interesting point about the value of following global rules in storage decisions compared to the intelligence gained from analysing historical data such as product trends. Instead of the rule that all products must be stored in the closest empty location for instance, it is more efficient for products that are usually retrieved together for the production line to be stored together. However, this information is dynamic and based on recent historical data.

Of the indirect measures of warehouse performance, labour is especially important since any inefficiency on the part of a human operator can impact shipping and delivery time. The importance of the human element (from operators to supervisors and management) as the actor whose "service failure or inefficient performance directly increases customer-order cycle time and negatively impacts the level of service as perceived by the customers" (Staudt, Alpan, Di Mascolo, \& Rodriguez, 2015) cannot be overlooked.

A different way of thinking of work environments is that of a cooperative partnership rather than a differentiation between the "computer system" and the "human" as entities that enhance the fulfilment of goals. The desired precepts of a system in which human and nonhuman agents interact in a "team" are that: 1) agents agree to collaborate to achieve a joint state and goal, 2) agents are predictable, able to be directed, observant and transparent in intentions and behaviour and 3) agents coordinate, negotiate and communicate (Klien, Woods, Bradshaw, Hoffman, \& Feltovich, 2004).

The design of Decision Support Systems (DSS) that can emulate human decision making would best be based on an understanding of human decision making but this is a complex psychological construct (Arnott \& Pervan, 2005). Firstly, humans are restricted by the availability and limits of cognitive resources, by biases and by time (Einhorn \& Hogarth, 1981). Nowadays, vast amounts of data can be stored and analysed. Information overload can stunt human decision making. DSS systems can assist humans most in the areas where they are most deficient: computational speed and planning (scenario generation) and it has been suggested that a human-machine collaboration is the best way to breach the gap between human limitations and computer understanding (Pohl, 1999). As warehouse operations become more complex, decision support tools that augment the ability of people to make appropriate and timely decisions has many advantages (Miller, 2018).

Real-time data capture supported by IoT, data mining advances and non-human agency converge on the concept of a digital twin first developed in Glaessgen and Stargel (2012). The digital twin is proposed to have a complete and individual representation of its non-digital counterpart. It uses available information from sensors and via data mining of other sources 
and mirrors the operating physical twin in order to predict the probability of future failure points based on hypothetical simulated scenarios.

A digital twin consists of three parts: physical product, virtual product, and connected data that tie the physical and virtual product space (Tao et al., 2018). The virtual product is a realtime reflection of the physical product and is being updated in real-time to maintain this mirroring. Connected data consists of various real-time and historical data that can be analysed and mined in a converged state (Tao et al., 2018). The connected data helps form a complete view of the state of an element and enhances decision-making.

In the model presented in this paper, new technologies and algorithms are used to augment the natural decision-making and learning capacity of people to create more flexible and productive work environments.

\section{Methodology}

Design Science research methodology (DSRM) was used for this study because of its iterative approach to developing and improving an IT artefact. An artefact is considered "any designed object in which a research contribution is embedded in the design" (Peffers, Tuunanen, Rothenberger, \& Chatterjee, 2007). Hevner, March, Park, and Ram (2004) describe design science research as "inherently, a problem-solving process". The seven guidelines for effective design science research established by Hevner et al. (2004) included designing a viable artifact, problem relevance, evaluation, contribution, research rigour, design as a search process and the communication of that research.

In this research, the artefact is a distributed WMS model tested via simulation. The six DSRM activities as defined by Peffers et al. (2007) were used as follows:

1. Identify Problem and Motivate. The problem addresses the real-time operational issues that cause bottlenecks and impede product flow in warehouses. As well as a literature review, semi-structured exploratory interviews were undertaken with warehouse professionals to confirm operational issues in warehouses. The results of these interviews are the subject of another paper.

2. Define Objectives of a Solution. Following the thematic analysis of the exploratory interviews, the data was examined to determine the link to the problem domain and the measures that would be used to evaluate the artefact that would be developed. The determined objective was to develop a model for designing a WMS that makes use of distributed intelligence. This model provides a way to reorganise around real-time constraints before they become bottlenecks and augment human decision-making and operational control.

3. Design and Development. This involved the requirements analysis, the design of a conceptual model, agent model, agent communication protocol, state diagrams, simulation development and the development of evaluation scenarios and measures. This step is the main focus of this paper.

4. Demonstration. The developed model was tested in the simulation using generated data (locations, products, inventory, orders etc.) against standard WMS algorithms. 
5. Evaluation. This activity involves determining how well the artefact matches the problem solution defined in activity 2. The simulation environment that was designed and constructed was used to evaluate the feasibility of the WMS design framework developed.

6. Communication. This paper is communicating the WMS design framework developed and evaluated in this study. We hope to make all phases and results of this study available through publication.

Three distinct design iterations are described below. These comprised the design and development activity of the DSRM methodology and were iteratively modified following demonstration/testing activities.

1. Development of the conceptual model (depicted in Figure 1). This involved defining the industry problem and reviewing the literature. The conceptual model was developed via a synthesis of research literature and industry experience.

2. Design of the multi-agent system. This involved defining and specifying the types of agents required, their specifications, goals and behaviours. This is elaborated in section 4 .

3. Simulation. This involved developing a warehouse simulation environment and coding the behaviour of the agents, using JadeX. The simulation was used to validate the design of the conceptual model. Real-world scenarios and constraints were used to test and evaluate the model. This is elaborated in section 5 .

\section{Conceptual Design Overview}

The design development of a distributed framework for a warehouse management system involved: Requirements Modelling and Agent Modelling. Requirements analysis was used to determine the required agents and their interactions. Agent modelling determined the agent states, goals, plans and modes of interaction.

\subsection{Requirements Analysis}

ANEMONA (A Multi-agent Methodology for Holonic Manufacturing Systems) (Botti \& Giret, 2008) was used to develop a basic requirements analysis of the agent and agent responsibilities for a warehouse. ANEMONA was chosen because of the structured and iterative approach to analysing and defining agents from the organisational diagram to abstract agents, goals, tasks and interactions into agents. Figure 2 shows a version of the abstract agents representing various warehouse roles. 


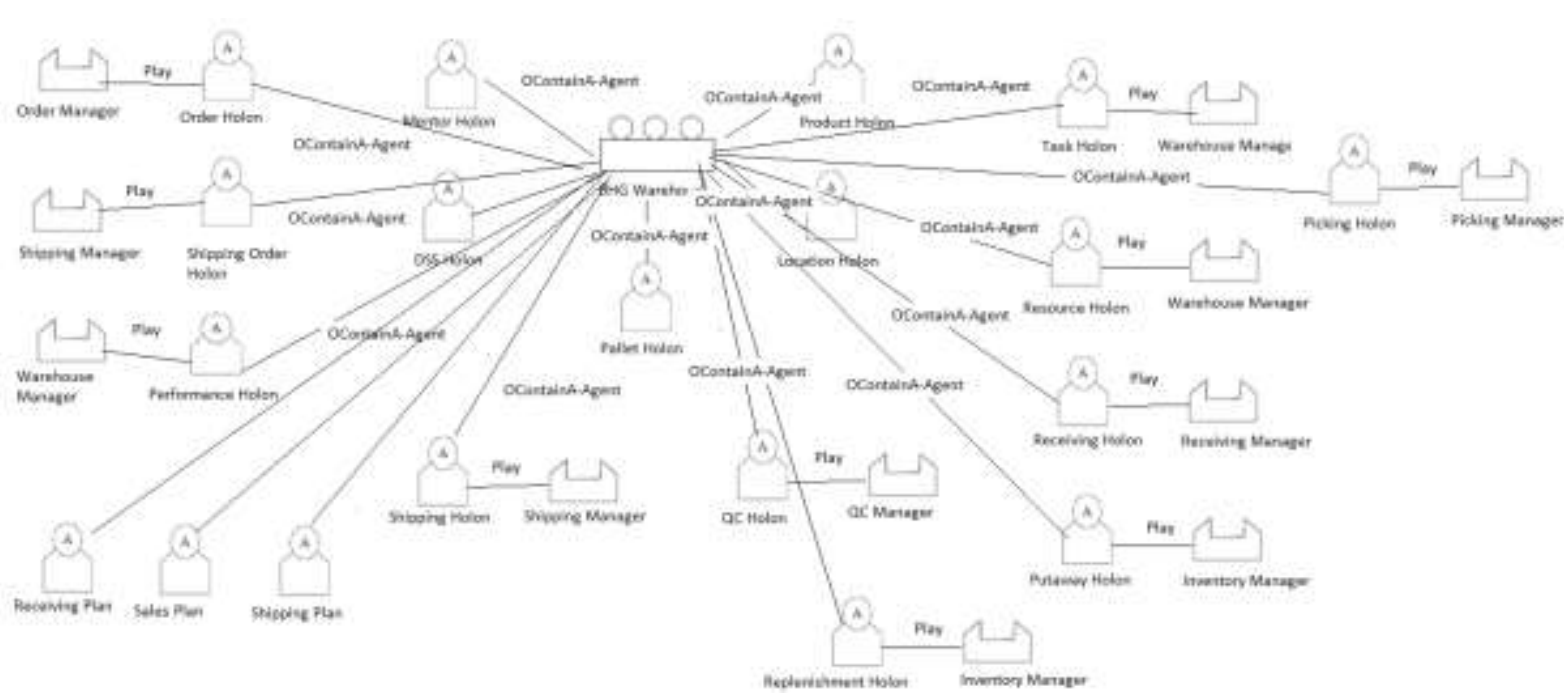

Figure 2 Abstract agents and the organisational diagram

\subsection{The Agent Model}

Three types of agents were modelled: entity agents that were connected to a physical entity (location, inventory pallet, human, forklift, shipping pallet), mentor agents (forklift operator and selector operator mentors) and service agents. Entity agents could be associated with sensors and other direct feedback mechanisms. Mentor agents were associated with a human operator and were in control of communicating with other agents to obtain available tasks and communicating task instructions to the human operator. Service agents were either providers of a function or information or fulfilled a role (generally a manager role).

The design of the agents involved goals and plans that were either triggered by a request from another agent via a communication protocol or a change in the state of the agent due to a change in the environment. As such, the design of the agents involved state diagrams and an inter-agent communication protocol. Each agent had a defined communication interface through which they receive a request for information or action. Each communication protocol involved the exchange (incoming and outgoing) of a message (request or response) and an object of data. Figure 3 shows some of the agent interactions in a mostly non-hierarchical configuration.

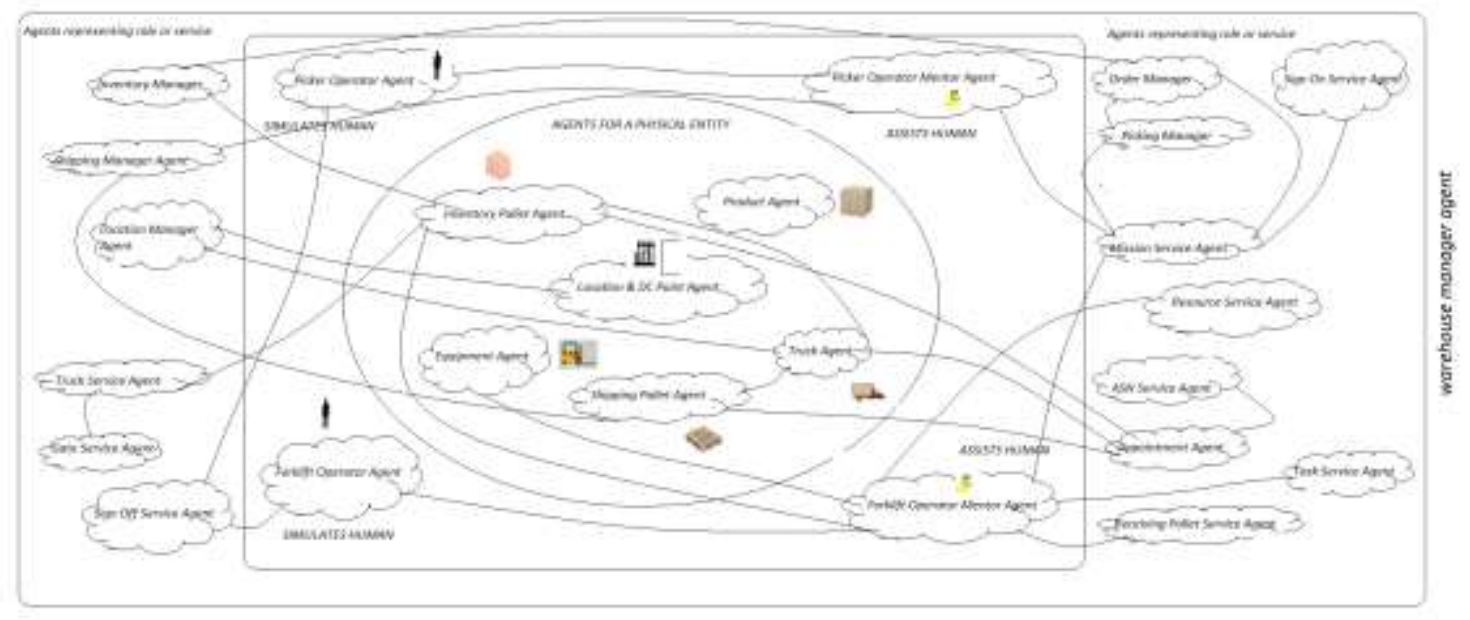

Figure 3 Agent Interactions 


\subsubsection{State Diagrams}

The agent goals were either triggered by a change of agent state or by a request from another agent. A change in state indicated a change in the environment and hence a change in the actions required from the agent. In the example of a receiving pallet in Figure 4, a change of state to "Arrived" triggers a goal to unload the pallet from the truck, creating the task required to unload it. This task is prioritised by the Work Priorities agent and allocated to a forklift operator through the task service agent. Once the pallet has been unloaded from the truck its state changes to "Unloaded" triggering a goal to find a suitable warehouse location for it. The goals that are initiated by a change of state in an agent are predictable. Entity agents maintain a state (status) and a position within the warehouse so that this can be maintained when the simulation is restarted.

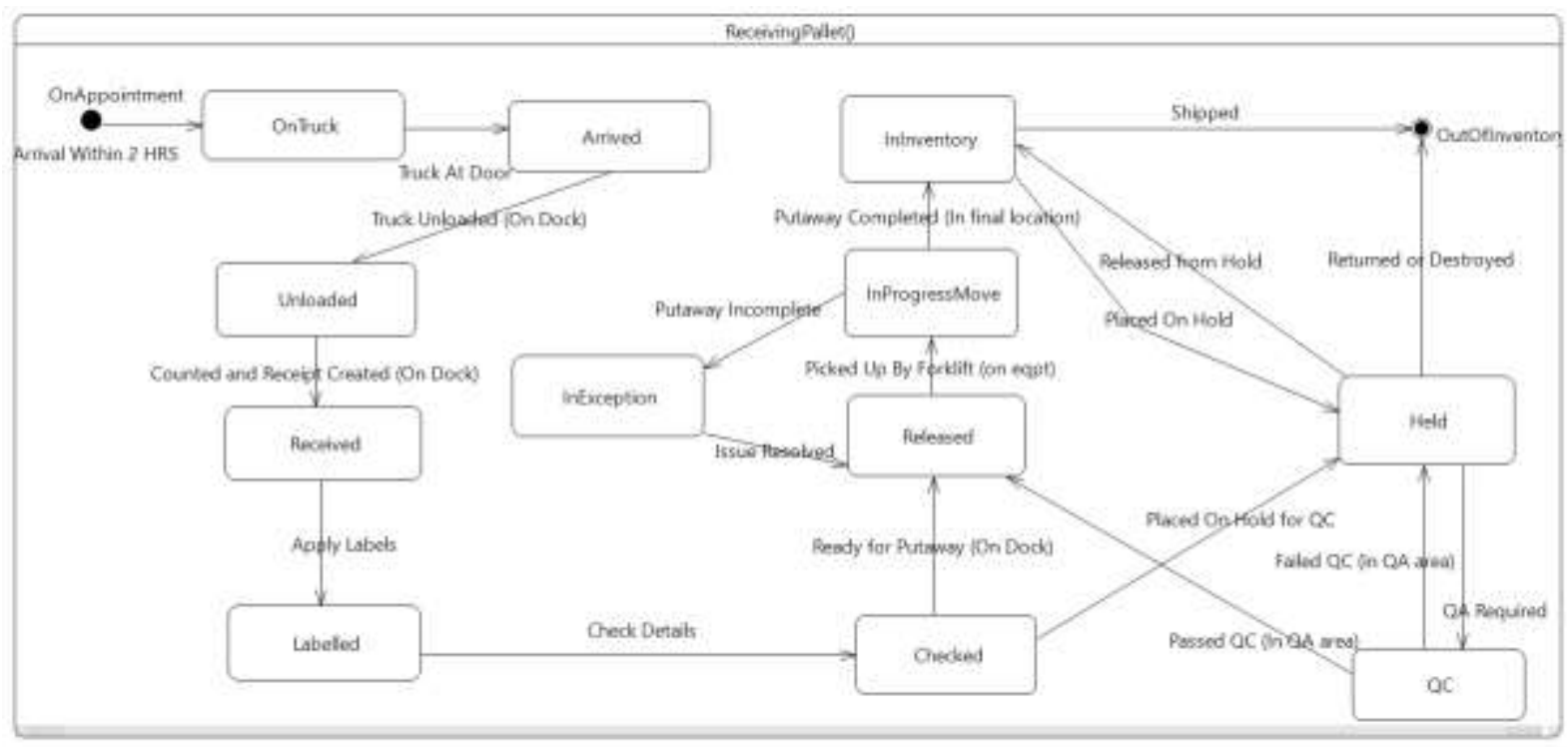

Figure 4 State Diagram for a pallet received into the warehouse

The entity agents such as the receiving pallet agent above were paired with inanimate objects to which agency is not usually ascribed. All entity agents had a real-time vision of other entity agents in their shared environment. Within the simulation this could be set to the entire environment or to a more realistic proximal distance. Granting agency to inanimate objects means that they are not just represented as a number within a WMS that are acted on but have the ability to determine their required action based on the current state. Another example of this is the location or point (staging, door, equipment etc.) entity agent (Figure 5). When the receiving pallet agent requests a storage location for itself, the location manager service may use any suitable algorithm which may change based on the current state of the environment. When a suitable location for put-away is found, the location agent must agree to allocate. This is a point at which the location agent, having more accurate information about its own state and the state of its surroundings may refuse the allocation thus preventing an operational exception from occurring when the forklift driver arrives at that location with the receiving pallet. Entity agents are a form of digital twin albeit a not very complicated one and without analytical capabilities. 


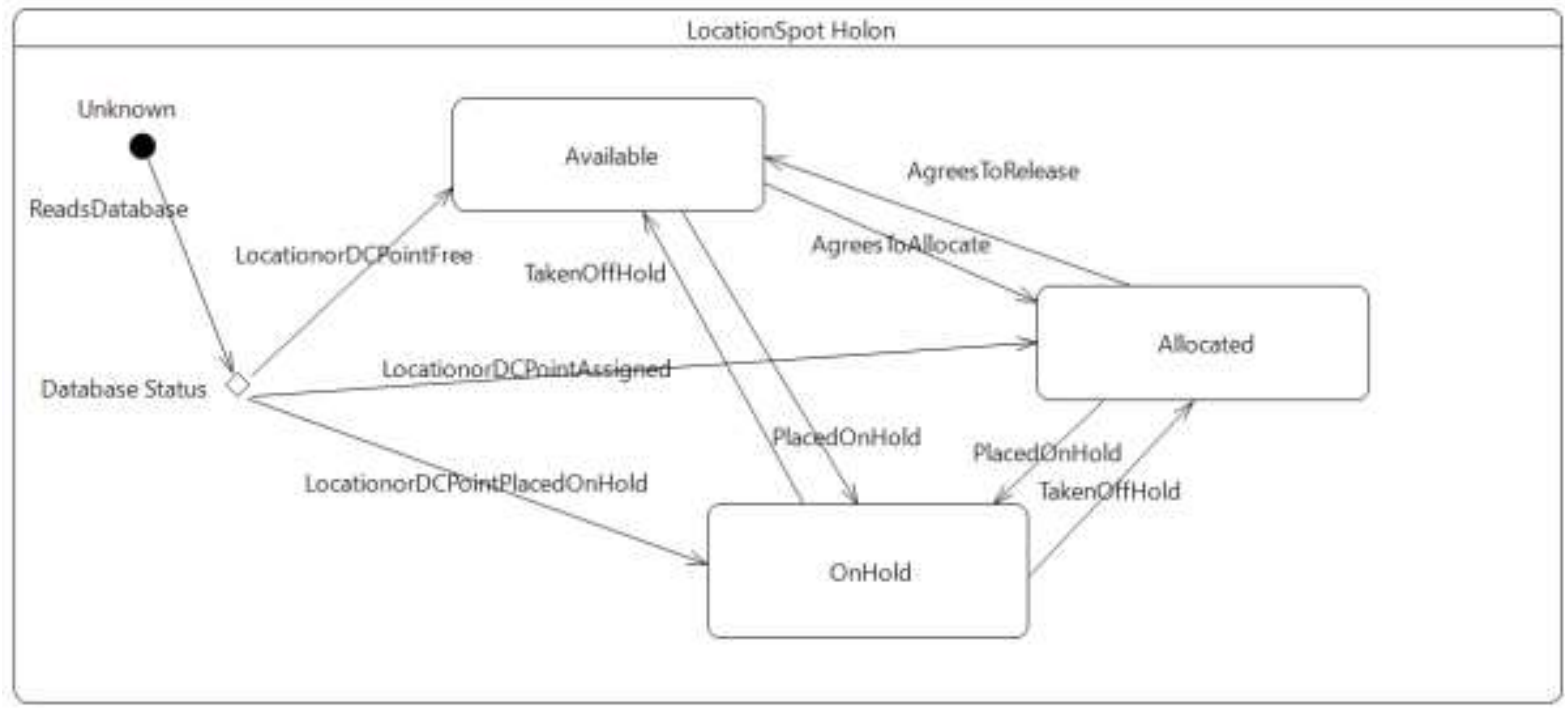

Figure 5 State Diagram for a Location Spot Entity Agent

The mentor agents that are associated to a human agent are more complex. The mentor agent mirrors the state of their human operator counterpart who is constantly moving within their environment, unlike the physical objects paired with an entity agent. The mentor agents (Forklift Operator Mentor and Picker Operator Mentor) have the ability to re-arrange the order of the task allocation based on environment information such as congestion, to release a task to be completed by another operator or to signal a review of similar tasks once it is determined that the current task cannot be completed. For example, when the picker operator mentor agent (Figure 6) detects a short pick at a primary selection location, the primary inventory pallet will request a replenishment pallet. If there are none available and none expected into the warehouse, then the picking manager service agent is signalled and it will either move or invalidate subsequent pick tasks from the same location preventing unnecessary work that cannot be completed.

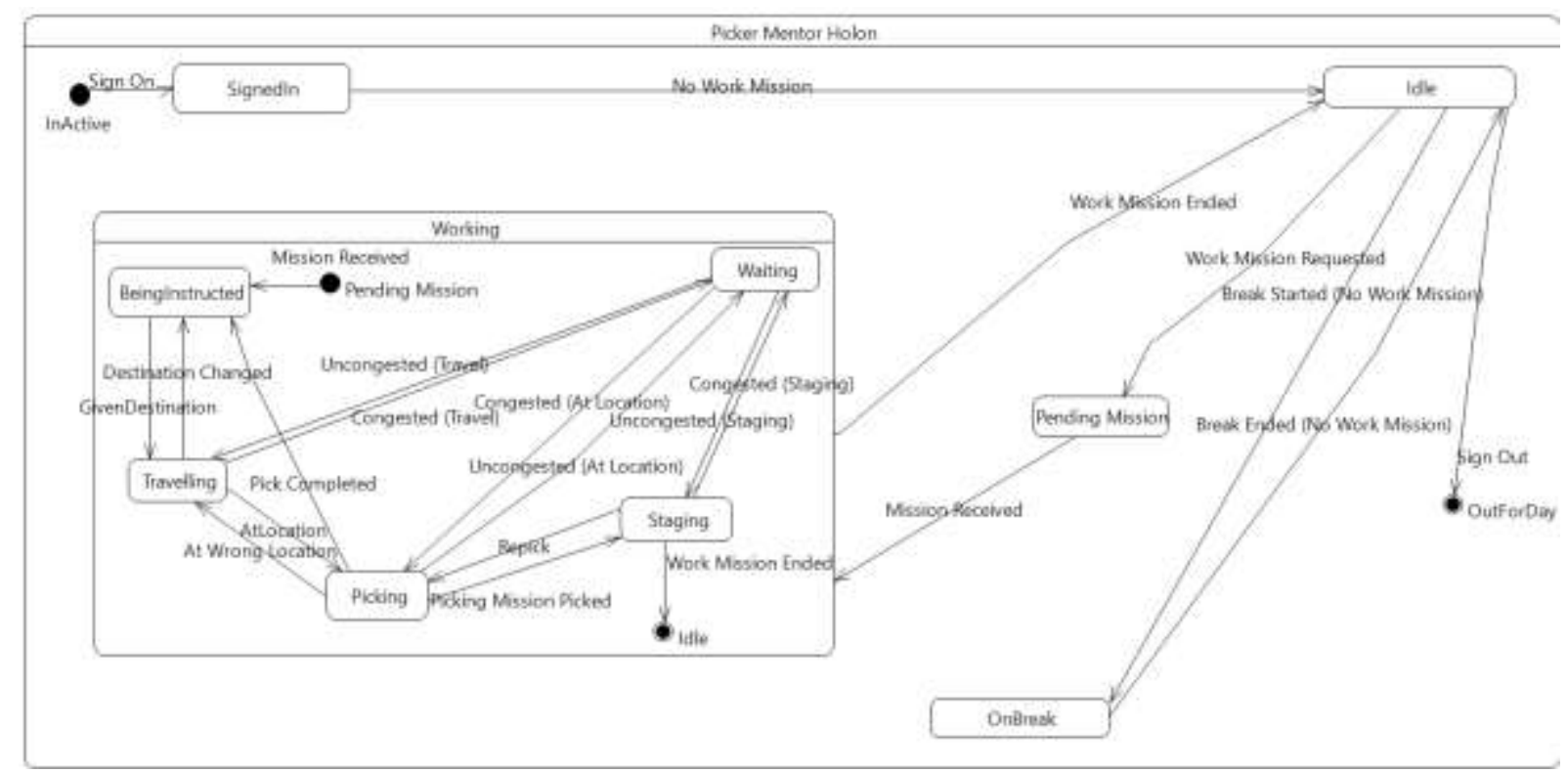

Figure 6 Picker Operator Mentor Agent State Diagram 


\subsubsection{Communication Protocol}

Each agent has a defined communication interface through which they receive a request for information or action. Each communication protocol involved the exchange (incoming and outgoing) of a message (request or response) and an object of data.

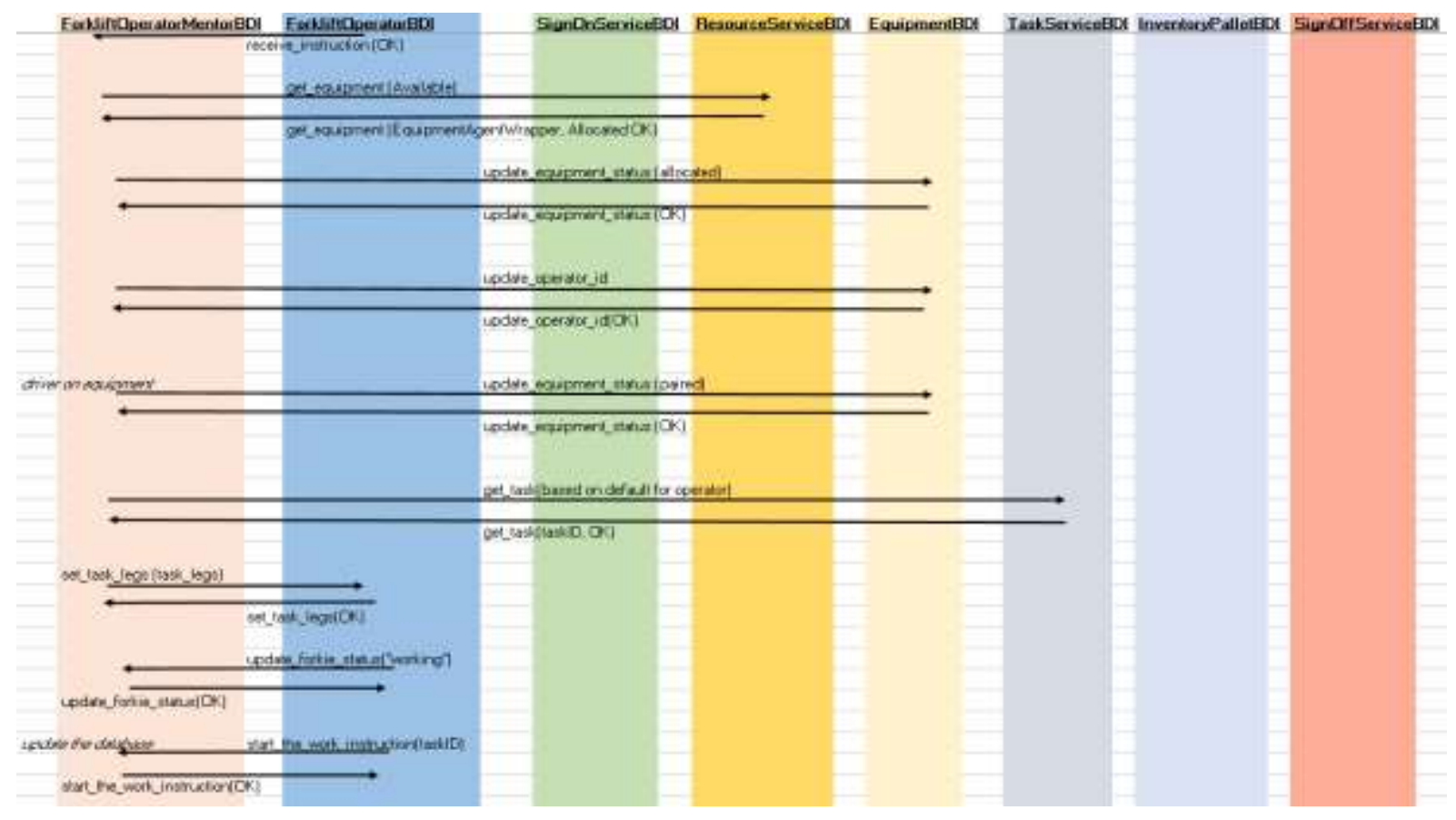

Figure 7 Communication of Forklift Operator Mentor

The communication between agents does not follow a strict hierarchical configuration unless a request was required to be directed to a manager service agent instead of an entity agent. For instance, a picker mentor agent could not request another picker mentor agent to alter the path of its mission directly. This request had to be made to a picker manager service. Figure 7 shows an excerpt of the communication initiated from the forklift mentor agent and other agents when the forklift operator signs on for their shift. The communication between the mentor and the human operator is a two-way communication whereby a request is issued by the mentor and accepted or completed by the operator. In a real-life implementation this communication would be via a voice headset or via an RF screen or hand-held device.

\subsection{Service Agents - Provision for plug-ins}

Service agents have the greatest scope for including rules surmised from the data mining of historical data. Since they receive real-time requests, instead of following one rule or algorithm for responding to a request, such as a request for a forklift work task or a selection mission, it may adjust its rules based on the state of the environment. The state of the environment can include time of the day, current number of operators, the number of available equipment - all of which equate to the capacity of the warehouse, versus the demand on the warehouse (orders).

For instance, the work priorities service agent receives requests to review the priorities of work tasks from other agents based on some operational event. These requests are received into a queue. When the warehouse capacity is under pressure, the priorities for task allocation become more important so the queue (Figure 8 and Figure 9) itself can be re-ordered based on 
the number of requests for a particular product, the number of orders requiring that product and the expected departure time of the orders with those same products for instance.

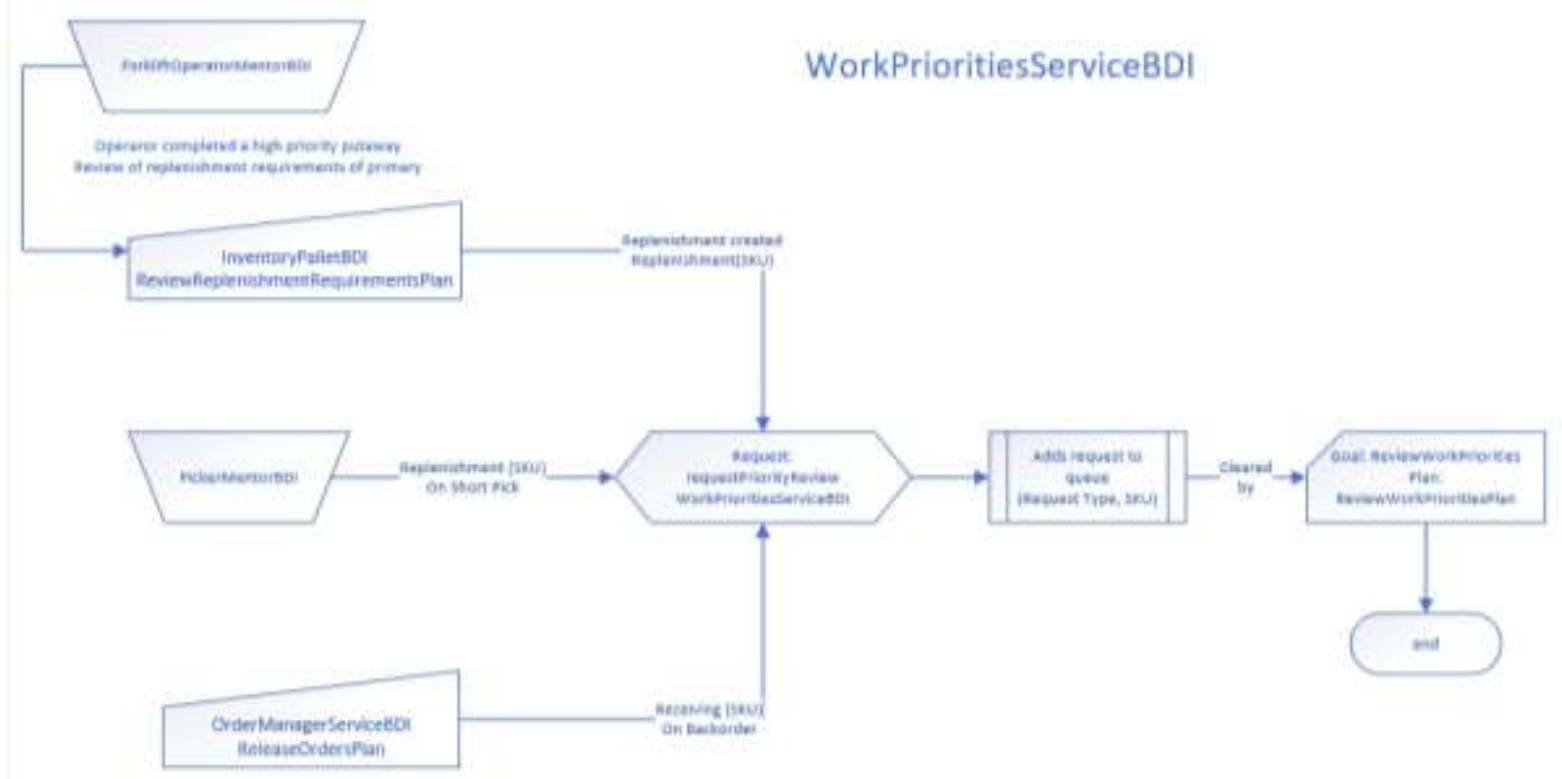

Figure 8 Work Priorities Service Agent

Multiple requests for priority reviews for the same product beyond a threshold (from machine learning) can signal a potential issue to a human operator and assist in the decision-making process for resolving the issue with potential solution scenarios. This form of DSS involves human-machine collaboration. It is not necessarily always up to the human decision-maker to identify potential issues with time-consuming reports but it may be more effective to allow a human decision-maker to decide the best course of action based on scenarios generated by the DSS and by critical evaluation of the situation.

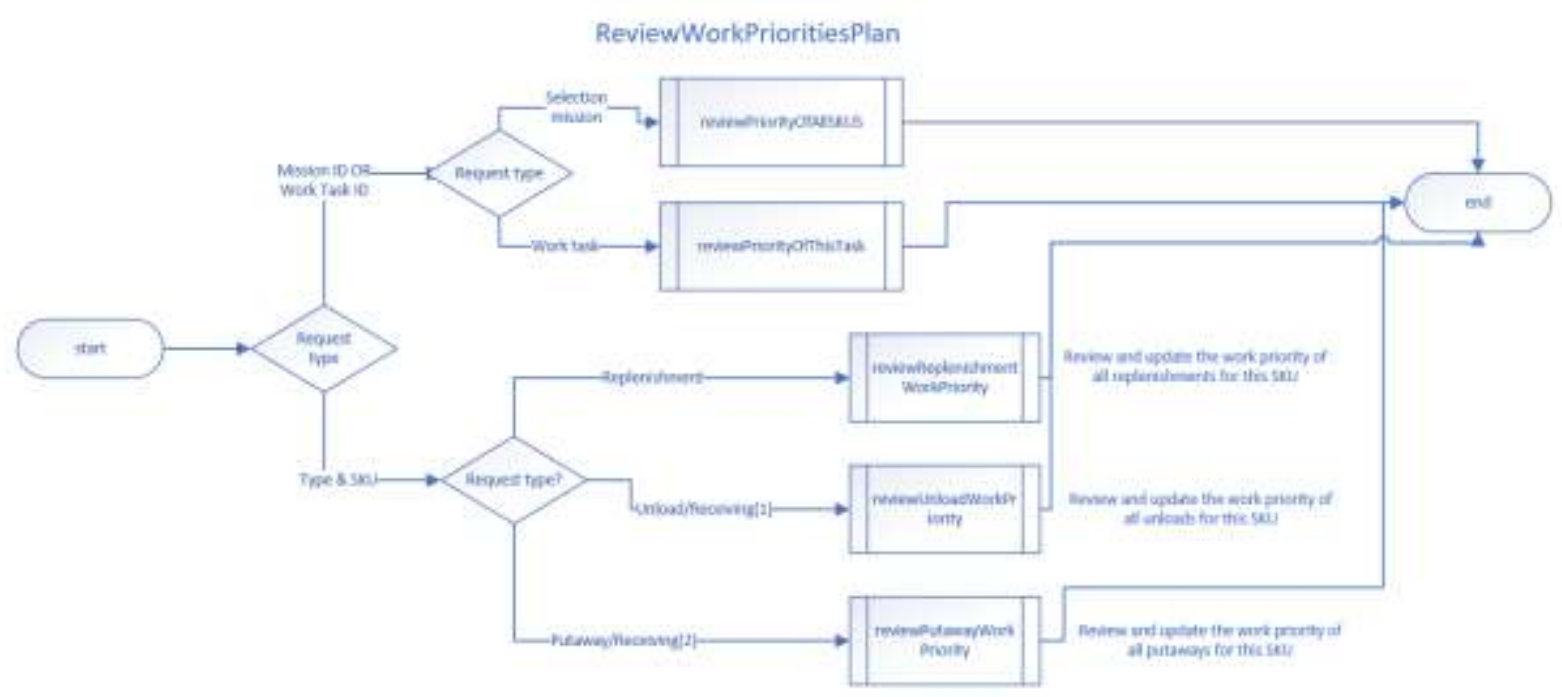

Figure 9 Work Priorities Service agent actioning requests

Another example of this is the inventory pallet agent determining its replenishment requirements. It will use time estimates from the Picker Manager Service for the "time until empty" and from the Inventory Service Manager for "time until replenishment can be 
completed". Both these estimates can be based on the current environment instead of stored averages so that the replenishment is created and allocated in time (Figure 10).

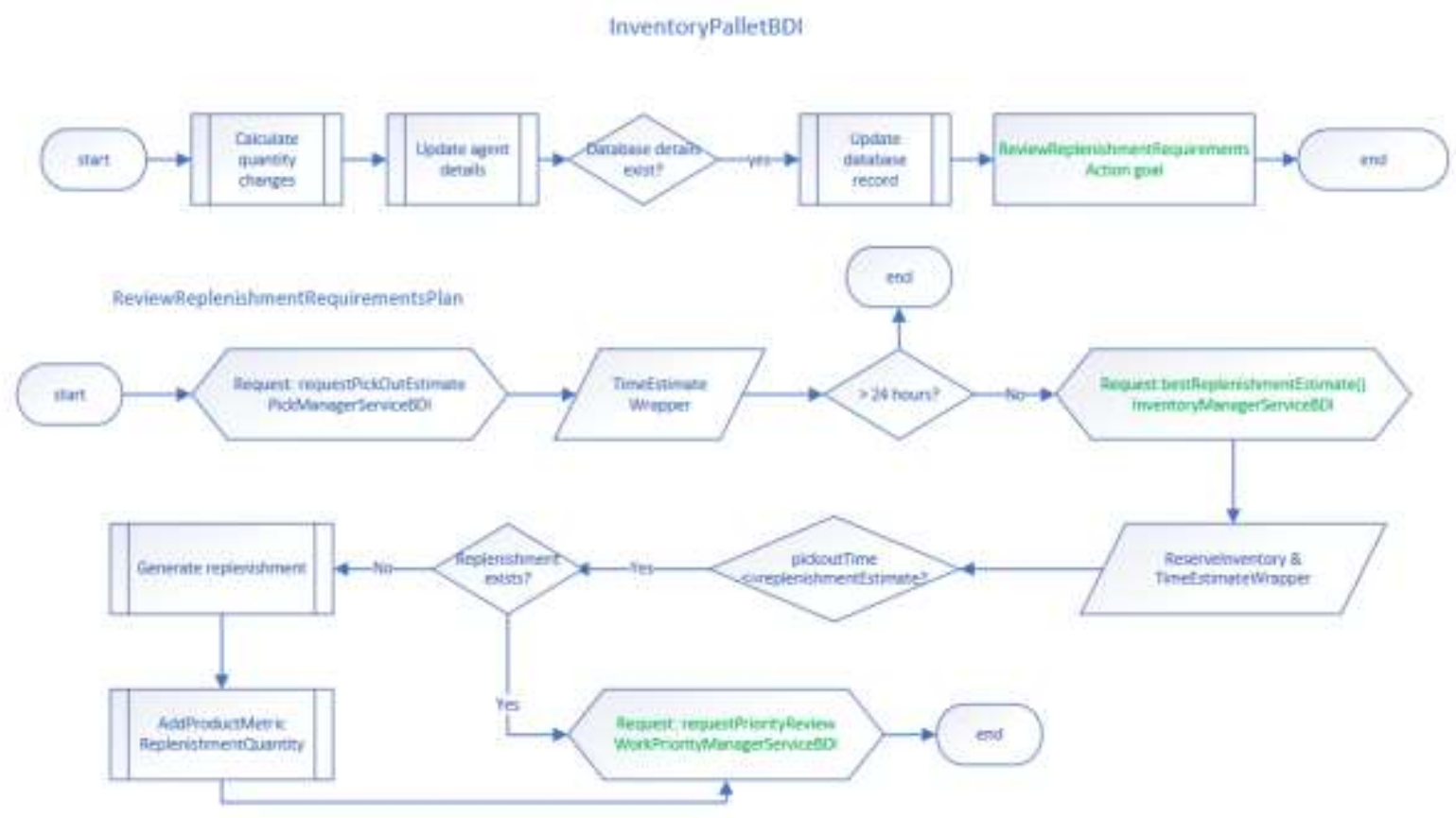

Figure 10 Primary Selection Inventory Pallet reviewing replenishment requirements

\section{Simulation Development}

This research examined various tools for the simulation development (Bordini, Dastani, Dix, \& Seghrouchni, 2009). The tool had to be open-source and programmable in a language that was already familiar to the researchers. The agent programming tool had to support BDI (Belief, Desire, Intentions) agents where a goal could be achieved through different plans that were deployed based on the real-time environment. JADEX (Pokahr, Braubach, \& Lamersdorf, 2005) was chosen and used to develop the agent simulation with associated database tables using MySql (Widenius, Axmark, \& Arno, 2002). JADEX agents are based on the traditional BDI agent model and its active component features facilitate the interactions between agents. The open-source multi-agent platform tools considered for the development of this simulation were Jason (Bordini, Hübner, \& Wooldridge, 2007), JADE (Bellifemine, Caire, \& Greenwood, 2007) and JADEX. Jason is an interpreter for an extended version of the logic-based language of AgentSpeak. JADE did not have BDI agent infrastructure, and this was provided by JADEX. Although all three were trialled, JADEX was chosen for its perceived ease of use and the availability of resources for supporting the development of the simulation.

\subsection{Simulation Scenarios}

Three common warehouse scenarios were selected to demonstrate the utility of the agent model in simulation. These are:

Scenario 1-Generating a Replenishment. This scenario is based on the precept that inventory received into the warehouse should be checked and stored in a sequence that prioritises stock that will be required sooner based on order demand. This is often a manual process in warehouse systems that are increasingly reliant on "just in time" inventory. The visibility of incoming goods required for order fulfilment in real-time, discerning and updating the 
priorities of the receipt of these goods in order to avoid an exception in picking is demonstrated in this scenario.

Scenario 2 - Short Pick in Selection. Order selection is still the most costly warehouse task performed and travel time in order selection is considered a major and "unproductive" component. Extant research focuses on optimising pick paths to minimise travel time. This scenario demonstrates minimising wasted travel time by avoiding short-picks in selection when an exception is detected which means that the intended selection tasks will not be successful.

Scenario 3 - Congestion in selection. Congestion occurs in real-time and hence is difficult to predict and is largely left up to the selectors to manage using the available options at their disposal - generally "skip" the pick or "wait". Congestion is also generally not tracked for analysis. This scenario demonstrates minimising unproductive time in selection due to congestion and the ability to track congestion and the success of congestion avoidance scenarios.

\subsubsection{Simulation Scenario 1 - Generating a Replenishment}

A replenishment is the refill of a primary selection location with product from another location. It involves identifying that there is a shortage in the primary selection location and triggering the move required to fill it in enough time to prevent shortages during order selection. The scenario is as follows:

- $\quad$ A customer order for 4 of product 108081 arrives into the system. The selection location for product 108081 is "01-01-1-1" and only has a quantity of 3. A pallet of 40 cases for product 108081 is on a receiving truck that has arrived with 10 other pallets but has not been unloaded yet. Table 1 shows the sequence of events in the distributed model in simulation.

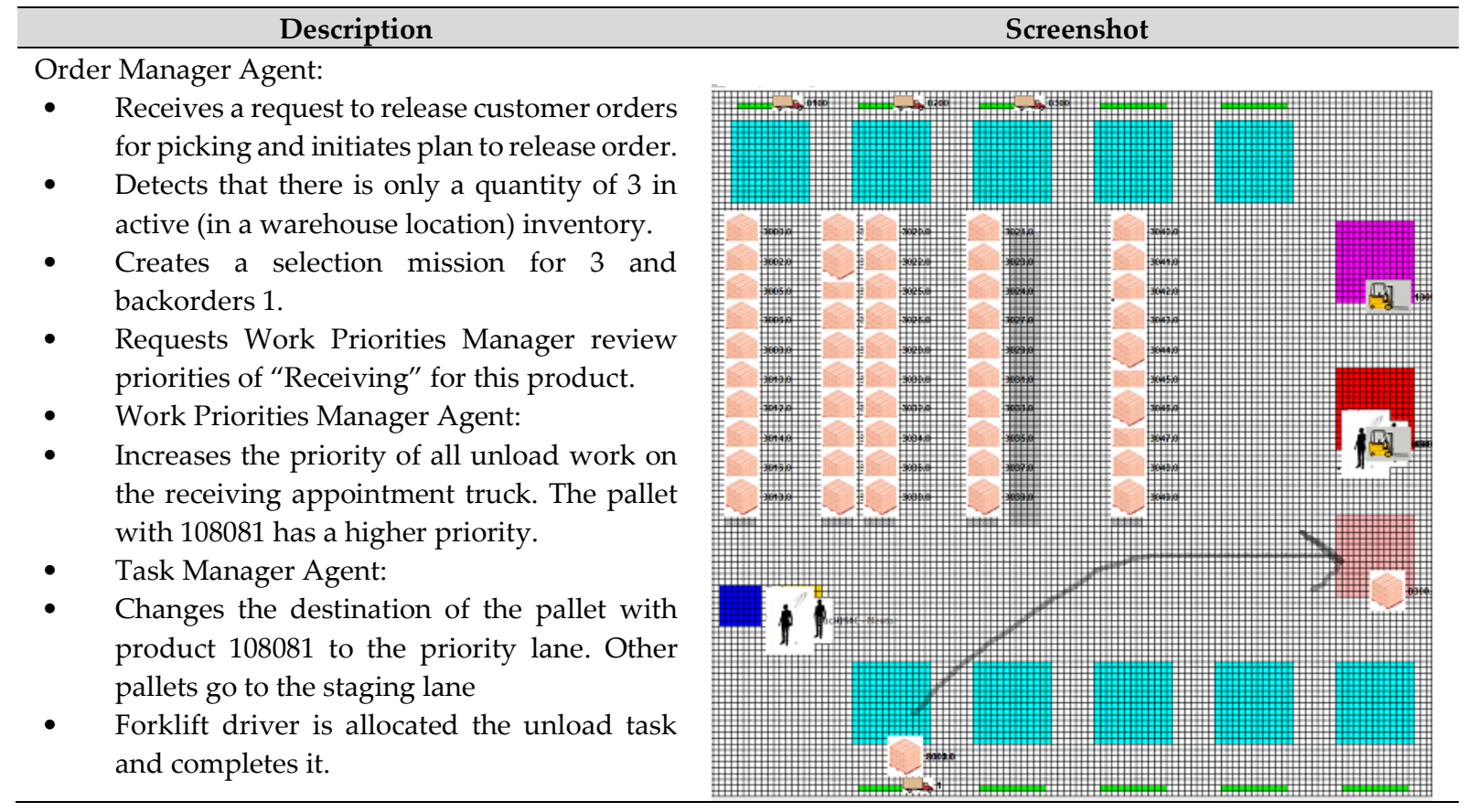




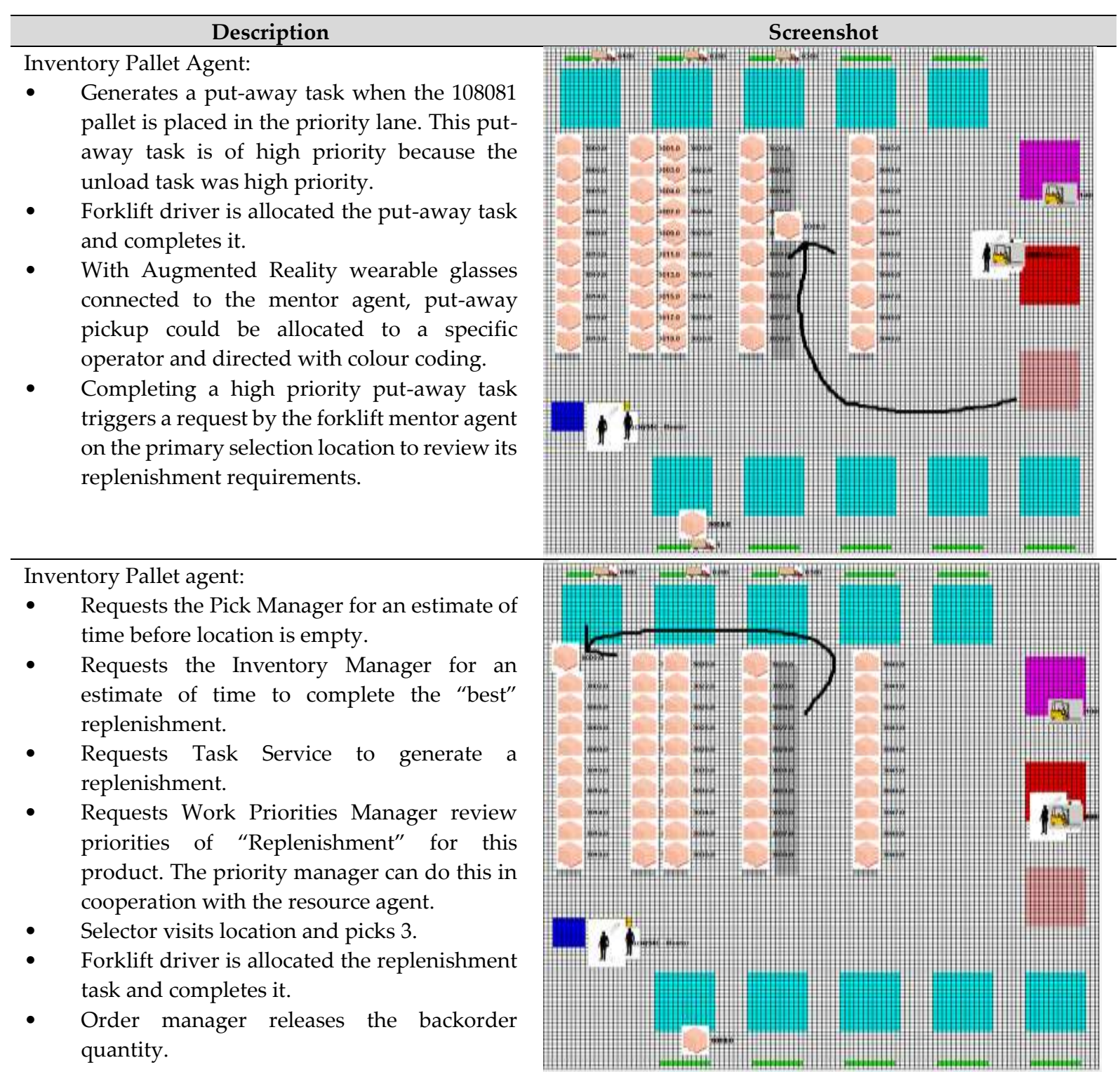

Table 1 Distributed Model for Replenishment Scenario

\subsubsection{Simulation Scenario 2 - Short Pick in Selection}

There has been a promotion for end of model product that has caused a spike in demand for that product. The warehouse expected to have enough in stock to meet the projected demand but an error in master data (the number of products on a supplier pallet) has meant that the warehouse has overestimated stock levels. The scenario is as follows:

- $\quad$ Four customer orders for 4 each of product 108081 arrive into the system. The system indicates that the selection location for product 108081 is "01-01-1-1" and has 30 but actually only has a quantity of 10 . There are no available pallets in reserve for this product. Table 2 shows the sequence of events in the distributed model in simulation.

\section{Description}

Order Manager Agent:

- $\quad$ Receives a request to release customer orders for picking and initiates plan to release orders.

- Erroneously detects that there is a quantity of 30 in active (in a warehouse location) inventory.

- Creates 5 selection missions for this product along with other products ordered by the customer. 
Description

Picking Manager Service:

- $\quad$ Receives a request from the Mission Service to make selection missions available.

- $\quad$ Makes all 5 selection missions available for pickers.

Mission Service agent:

- $\quad$ Allocates missions to picker mentor agent for associated picker.

Pick Operator Agent/Picker Mentor Agent

- $\quad$ First 2 selection missions complete successfully.

Inventory Pallet Agent:

- $\quad$ Requests replenishment but there are no available reserve pallets.

- Informs Picking Manager of anticipated short pick issue.

Pick Operator Agent/Picker Mentor Agent

- $\quad$ Picker Operator travels to selection location, picks remaining 2. Two are not picked (shorted).

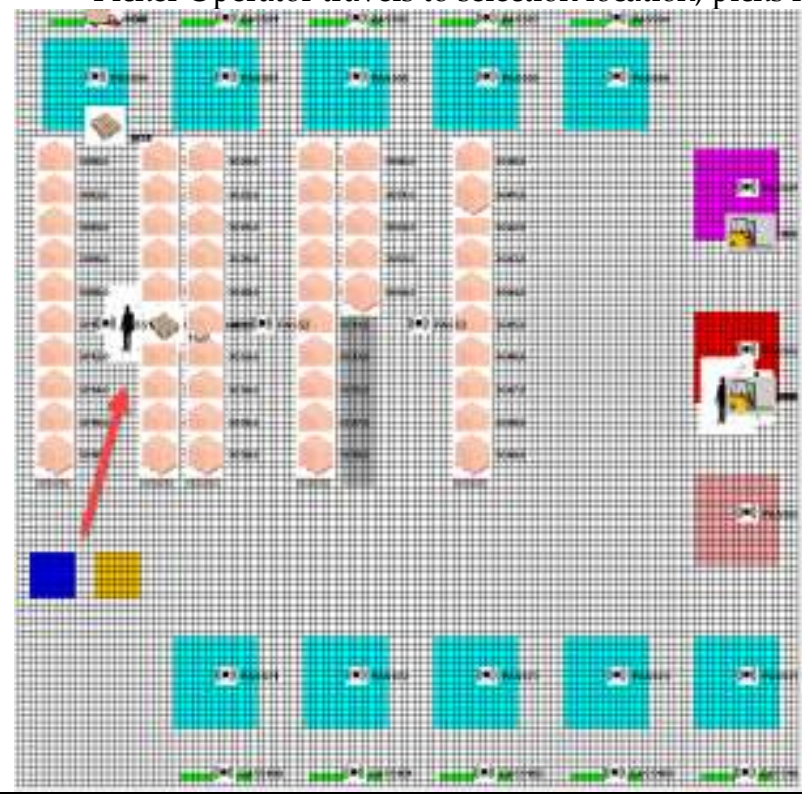

Inventory Pallet Agent:

- Updates balance in location to zero.

- $\quad$ Requests replenishment but there are no available reserve pallets.

- Informs Picking Manager of actual short pick issue.

Picking Manager

- Modifies available but as yet unallocated selection missions to remove this pick instruction. Selection mission cancelled if no other pick instructions.

- $\quad$ Requests order manager update orders and backorder the items not picked.

- $\quad$ Notifies warehouse manager agent of issue.

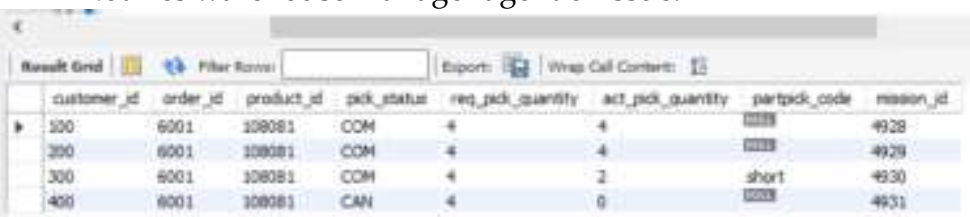

Warehouse Manager Service Agent

- $\quad$ Sends issue to inventory controller mentor agent.

Inventory Controller Mentor Agent

- Displays issue and potential scenarios to inventory controller human agent

Table 2 Distributed Scenario for short pick in selection 


\subsubsection{Simulation Scenario 3 - Congestion in Selection}

There has been a promotion for end of model product that has caused a spike in demand for that product. The warehouse does have enough in stock to meet the projected demand. The scenario is as follows:

- $\quad$ Forty customer orders for 1 each of product 108081 arrive into the system. The system indicates that the selection location for product 108081 is "01-01-1-1" and has 50. Table 3 shows the sequence of events in the distributed model in simulation.

\begin{tabular}{|l|l|}
\hline & Description \\
\hline Order Manager Agent: \\
Receives a request to release customer orders for picking and initiates plan to release orders. \\
- Creates 40 selection missions for this product along with other products ordered by the customer. \\
\hline $\begin{array}{l}\text { Picking Manager Service: } \\
\text { Receives a request from the Mission Service to make selection missions available. } \\
\text { Makes 40 selection missions available for pickers. }\end{array}$ \\
\hline $\begin{array}{l}\text { Mission Service agent: } \\
\text { Allocates missions to picker mentor agent for associated picker. }\end{array}$ \\
\hline $\begin{array}{l}\text { Pick Operator Agent/Picker Mentor Agent } \\
\text { Requests status from Passage Agent (sensor in the aisle for the next selection location to be picked) }\end{array}$ \\
\hline $\begin{array}{l}\text { Passage Agent } \\
\text { Picker Mentor Agent: }\end{array}$ \\
\hline
\end{tabular}

Table 3 Distributed Scenario for Congestion in Selection

\subsection{Simulation Evaluation}

These scenarios as described above with the agent model interaction were reviewed by an expert from the field with 25 years of experience in warehousing and distribution. All three scenarios were evaluated as realistic. Feedback on scenario one related to directing the 
putaway of priority pallets directly to the selection location from priority staging instead of via reserve inventory, and has been incorporated into a future test scenario.

\section{Discussion}

Warehouses and warehouse management systems are complex and dynamic systems traditionally controlled by strict adherence to process and sequence. The problem with this is that when an unexpected exception occurs, the process cannot be followed and something else has to be done in its place and this causes delays and flow-on effects. Adaptive mechatronic systems with societies of autonomous adaptive agents can exhibit self-organising behaviours that can overcome these small bottlenecks in operational processing. As in the scenario in section 5, the pallet yet to be unloaded can be prioritised and the task destination changed dynamically by the real-time detection of a deficit in selection. Adaptation can occur by recording predicted estimates of task completion against actual task completion outcome and incorporating the error (difference) into future estimates. Adaptation can also occur in storage by connecting patterns of inventory inaccuracies with product characteristics such as similarity contributing to operator error.

Agents can communicate a real-time status. This means that dynamic tasks can be generated closer to the time that they will be actioned. As a result, more appropriate locations will be available for inventory to be put away, the resources to complete the tasks will be available (potentially) and time and productivity is not spent actioning tasks that are not ready to be completed and that may potentially not be able to be completed.

Agents have goals and goals can persist. Agents continue to execute plans until the goal is fulfilled so an exception does not need to be handled manually all the time. For instance, a replenishment that failed to be physically completed will trigger a replacement task (until the selection location is refilled). A pallet that failed to be physically put away in its designated location will be taken to the resolution zone and if appropriate will self-generate another put away task for itself. Tracking the reason for task failure (exceptions) in real-time allows more information to be given to the inventory manager to resolve issues that could not be selfresolved, aiding the decision-making process. Human intervention, where needed, can correct the system data to the point where the agents can continue their normal processing.

The purpose of the mentor agent is to provide a real-time ability to react to real-time changes in the environment. Human operators are generally just following instructions and encountering a real-time constraint issue such as congestion. The mentor agent, communicating agent-agent, agent-human or human-agent, can provide the advantage of a smarter view of the warehouse environment for the operator. With sensors detecting changes in the warehouse environment, different algorithms can be deployed to determine selector travel path or to re-evaluate the sequence of available tasks.

In a working warehouse environment this simulation could be run with actual data and could be used to enact scenarios which have caused an operational exception that was pre-emptively avoided to determine how the operational exception could happen again and become a failure point that is not managed to be avoided (increasing demand, increasing congestion, decreasing staff and equipment). Alternatively, scenarios that were a failure point can be used in the simulation to determine corrective action or pre-emptive detection that can be used to avoid the failure. 
The increase in e-commerce and omni-channel commerce has been putting a strain on warehouses and WMS systems. Many warehouse management systems (WMS) are not designed for this new way of working. In addition, warehouses by their design run efficiently when predicted demand is adequately met by warehouse capacity (goods, people and resources) and when operational procedures are clear and stringently followed. However, there are a number of small operational exceptions that can impede this smooth product flow and manifest as disruptions if not detected and mitigated early.

Past warehouse research literature has largely focused on optimisation strategies (pick paths, product storage and human ergonomic aspects). More recently research has begun to focus on realising a living or "smart" warehouse by giving inanimate objects properties and agency in order to respond faster to real-time events. It seems that this is a unique point in time, with emerging technologies such as data mining and analysis, sensor and IoT interconnectivity to create a WMS framework that is not just based on maintaining "standard" operations and their optimisation but also includes a basis of the real-time constraints that cause exceptions and lead to disruptions.

Estanjini et al. (2011) uses sensors and dynamic programming to calculate the most costeffective way to allocate the next forklift task which is smart optimisation. The intelligent product agent in Giannikas et al. (2013) seeks the best storage location each time it is received into the warehouse and this is based on a number of factors including "turnover rate, demand, the relationships with other products, the layout of the warehouse etc." This could also include minimisation of warehouse real-time exceptions. Giannikas et al. (2013) describes "unexpected events" such as "arrivals of new orders". In a warehouse environment there can also be many other unexpected events. The WMS agent in Trab et al. (2017) is based on real-time safety compatibility constraints of goods and people in a hazardous goods environment and is focused on minimising risk. Trab et al. (2017) describes an "unexpected event" as something that increases risk to "products, humans and the environment" such as "the presence of an empty product owing to a leak, and a human user without safety protection equipment".

In everyday warehouse operations, unexpected events pose a risk to efficiency and productivity. While the type of unexpected events may be known, the timeline of their occurrence and which operational exception will occur as a result is not known. For this reason, a WMS framework that can detect their occurrence and has built-in plans that can be deployed when they occur is needed both to mitigate flow-on risk and to provide real assistance to decision makers.

Current literature focuses on recording exceptions (Weissbach, Radmanu, \& Grabowski, 2009) and expediting individual exception resolution (McDonald Jr et al., 2018) but not on detecting an exception that is about to occur in order to avoid it, detecting exceptions in real-time, minimising the flow-on effect when an exception has occurred and augmenting the decisionmaking capacity of humans tasked with resolving these exceptions. This study proposes that incorporating these exceptions specifically into the WMS framework will make warehouses more able to respond to dynamic changes in the environment.

\section{Conclusion}

Warehouses are real-time environments that involve a work schedule, available resources, and time and space constraints. Increasingly, the outlook to solving this problem is to fully automate, however this may not be feasible for small to medium size warehouses or 
warehouse with specialist requirements. This research aims at innovation by augmenting people rather than replacing them. This paper presents a framework for designing warehouse management systems that uses distributed intelligence to react to real-time events and constraints, in order to mitigate the impact of small operational issues on product flow and order fulfilment. A simulation based on intelligent agents was designed and constructed in order to validate the viability of the framework.

The contribution of a new framework for WMS design is three-fold. Firstly, there are advantages to be gained in detecting warehouse exceptions in real-time before they escalate into disruptions. Although adherence to warehouse processes dictate smooth product flow, unexpected events can have a big impact on productivity and efficiency.

Secondly, academic research provides new algorithms and processes for optimisation and efficiency of warehouse processes, but these cannot be easily incorporated into current warehouse management systems. The design of service agents in this model can make adding new features such as the above more feasible in an environment where they can be used when applicable based on real-time environmental constraints and where their effectiveness can be evaluated in real-time and historically through data-mining.

Thirdly, decision support that supports the cognitive, memory and actual time constraints of the warehouse decision makers by providing packaged information about an exception and potential resolution scenarios facilitate the resolution of warehouse exceptions faster where a human decision-maker is required.

This paper contributes to industry by proposing a new way of designing warehouse management systems that augments and enhances the operations of manual warehouses instead of replacing them with automation. With advances in IoT and sensor technologies this approach may be more suitable to the challenges posed by demand being driven by the changing consumer behaviour.

Future research iterations of this project may take several paths. Various AI methods and algorithms can be tested as plug-ins within the intelligent agents, in the simulation, to improve operational functionality and the ability of the model to forecast and resolve problems. The metrics that will be used for evaluation purposes include stock out rate, orders shipped on time, shipping accuracy, queuing time and throughput. These metrics are based on the direct indicators of performance as defined by (Staudt et al., 2015). The number of operational exceptions that occur will also be measured for comparison.

\section{References}

Arnott, D., \& Pervan, G. (2005). A critical analysis of decision support systems research. Journal of information technology, 20(2), 67-87.

Barratt, M., Kull, T. J., \& Sodero, A. C. (2018). Inventory record inaccuracy dynamics and the role of employees within multi-channel distribution center inventory systems. Journal of Operations Management, 63, 6-24.

Bartholdi, J. J., \& Hackman, S. T. (2008). Warehouse \& Distribution Science: Release 0.89: Supply Chain and Logistics Institute.

Bellifemine, F. L., Caire, G., \& Greenwood, D. (2007). Developing multi-agent systems with JADE (Vol. 7): John Wiley \& Sons. 
Bordini, R. H., Dastani, M., Dix, J., \& Seghrouchni, A. E. F. (2009). Multi-Agent Programming: Springer.

Bordini, R. H., Hübner, J. F., \& Wooldridge, M. (2007). Programming multi-agent systems in AgentSpeak using Jason (Vol. 8): John Wiley \& Sons.

Botti, V., \& Giret, A. (2008). ANEMONA: A Mulit-agent Methodology for Holonic Manufacturing Systems. London: Springer London, London.

Davarzani, H., \& Norrman, A. (2015). Toward a relevant agenda for warehousing research: literature review and practitioners' input. Logistics Research, 8(1), 1.

De Koster, R., Le-Duc, T., \& Roodbergen, K. J. (2007). Design and control of warehouse order picking: A literature review. European Journal of Operational Research, 182(2), 481-501.

Ding, W. (2013). Study of smart warehouse management system based on the IOT. In intelligence computation and evolutionary computation (pp. 203-207): Springer.

Einhorn, H. J., \& Hogarth, R. M. (1981). Behavioral decision theory: Processes of judgement and choice. Annual review of psychology, 32(1), 53-88.

Estanjini, R. M., Lin, Y., Li, K., Guo, D., \& Paschalidis, I. C. (2011). Optimizing warehouse forklift dispatching using a sensor network and stochastic learning. IEEE Transactions on Industrial informatics, 7(3), 476-486.

García, A., Chang, Y., Abarca, A., \& Oh, C. (2007). RFID enhanced MAS for warehouse management. International Journal of Logistics Research and Applications, 10(2), 97-107. doi:10.1080/13675560701427379

Gharbi, S., Zgaya, H., \& Hammadi, S. (2013). Optimization of order picker path based on agent communication in warehouse logistics. IFAC Proceedings Volumes, 46(24), 7-14. doi:10.3182/20130911-3-BR-3021.00024

Giannikas, V., Lu, W., McFarlane, D., \& Hyde, J. (2013). Product intelligence in warehouse management: A case study. Paper presented at the International Conference on Industrial Applications of Holonic and Multi-Agent Systems.

Gibilaro, L., \& Mattarocci, G. (2019). The impact of corporate distress along the supply chain: evidences from United States. Supply Chain Management: An International Journal.

Glaessgen, E., \& Stargel, D. (2012). The digital twin paradigm for future NASA and US Air Force vehicles. Paper presented at the 53rd AIAA/ASME/ASCE/AHS/ASC structures, structural dynamics and materials conference 20th AIAA/ASME/AHS adaptive structures conference 14th AIAA.

Glock, C. H., Grosse, E. H., Elbert, R. M., \& Franzke, T. (2017). Maverick picking: the impact of modifications in work schedules on manual order picking processes. International Journal of Production Research, 55(21), 6344-6360.

Gu, J., Goetschalckx, M., \& McGinnis, L. F. (2007). Research on warehouse operation: A comprehensive review. European Journal of Operational Research, 177(1), 1-21.

Haneyah, S., Schutten, J. M., Schuur, P., \& Zijm, W. H. (2013). Generic planning and control of automated material handling systems: Practical requirements versus existing theory. Computers in Industry, 64(3), 177-190. 
Hevner, A. R., March, S. T., Park, J., \& Ram, S. (2004). Design Science in Information Systems Research. MIS Quarterly, 28(1), 75-105.

Karagiannaki, A., Papakiriakopoulos, D., \& Bardaki, C. (2011). Warehouse contextual factors affecting the impact of RFID. Industrial Management \& Data Systems, 111(5), 714-734.

Kim, B.-I., Graves, R., \& Heragu, S. (2002). Intelligent agent modeling of an industrial warehousing problem. IIE Transactions, 34(7), 601-612. doi:10.1080/07408170208928897

Klien, G., Woods, D. D., Bradshaw, J. M., Hoffman, R. R., \& Feltovich, P. J. (2004). Ten challenges for making automation a" team player" in joint human-agent activity. IEEE Intelligent Systems, 19(6), 91-95.

Latour, B. (2005). Reassembling the social an introduction to actor-network-theory. Oxford, New York: Oxford University Press.

Leung, K., Choy, K., Siu, P. K., Ho, G., Lam, H., \& Lee, C. K. (2018). A B2C e-commerce intelligent system for re-engineering the e-order fulfilment process. Expert Systems with Applications, 91, 386-401.

Liu, X. J., Liu, Q., Xu, W. J., Yang, L. W., Fan, L., \& Chen, B. (2013). Design and Implementation of Intelligent Warehousing Management System Using Internet of Things. Applied Mechanics and Materials, 432-432, 609-614. doi:10.4028/www.scientific.net/AMM.432.609

Lu, W., Giannikas, V., McFarlane, D., \& Hyde, J. (2014). The role of distributed intelligence in warehouse management systems. In Service orientation in holonic and multi-agent manufacturing and robotics (pp. 63-77): Springer.

Maka, A., Cupek, R., \& Wierzchanowski, M. (2011). Agent-based modeling for warehouse logistics systems. Paper presented at the 2011 UKSim 13th International Conference on Modelling and Simulation.

Marik, V., \& McFarlane, D. (2005). Industrial adoption of agent-based technologies. IEEE Intelligent Systems, 20(1), 27-35.

McDonald Jr, R. D., Agriel, F. L., Levi, E. S., Patel, V. N., Nanjanath, M., Madan, U., \& Glick, D. D. (2018). Utilizing automated aerial vehicles for transporting priority pick items. In: Google Patents.

McFarlane, D., Parlikad, A., Neely, A., \& Thorne, A. (2012). A Framework for Distributed Intelligent Automation Systems Developments. IFAC Proceedings Volumes, 45(6), 758-763. doi:10.3182/20120523-3-RO-2023.00325

Michel, R. (2016). Ready to Confront Complexity. Logistics Management (2002), 55(11), 6$48 \mathrm{~S}, 49 \mathrm{~S}, 50 \mathrm{~S}, 52 \mathrm{~S}, 54 \mathrm{~S}, 55 \mathrm{~S}$.

Michel, R. (2018a). 2018 Warehouse/DC Operations Survey: LABOR CRUNCH DRIVING AUTOMATION: The combined forces of a strong economy, e-commerce growth and a tight labor market are making it more important for distribution center operations to find ways to make their existing infrastructure and people more productive. Software and automation continue to prove to be a vital part of the solution. Modern Materials Handling, 73(11), 62.

Michel, R. (2018b). AUTOMATION \& ROBOTICS LEAD ROBUST OUTLOOK. Logistics Management (2002), 57(3), 40-47. 
Miller, S. (2018). AI: Augmentation, more so than automation. Asian Management Insights, 5(1), $1-20$.

Min, H. (2006). The applications of warehouse management systems: an exploratory study. International Journal of Logistics: Research and Applications, 9(2), 111-126.

Peffers, K., Tuunanen, T., Rothenberger, M. A., \& Chatterjee, S. (2007). A design science research methodology for information systems research. Journal of management information systems, 24(3), 45-77.

Pohl, J. G. (1999). Collaborative decision-support and the human-machine relationship. Collaborative Agent Design (CAD) Research Center, 18.

Pokahr, A., Braubach, L., \& Lamersdorf, W. (2005). Jadex: A BDI reasoning engine. In Multiagent programming (pp. 149-174): Springer.

Reaidy, P. J., Gunasekaran, A., \& Spalanzani, A. (2015). Bottom-up approach based on Internet of Things for order fulfillment in a collaborative warehousing environment. International Journal of Production Economics, 159, 29-40.

Richards, G. (2014). Warehouse Management : A Complete Guide to Improving Efficiency and Minimizing Costs in the Modern Warehouse (2nd Edition). London: London, GBR: Kogan Page.

Rouwenhorst, B., Reuter, B., Stockrahm, V., van Houtum, G.-J., Mantel, R., \& Zijm, W. H. (2000). Warehouse design and control: Framework and literature review. European Journal of Operational Research, 122(3), 515-533.

Rubrico, J. I. U., Ota, J., Higashi, T., \& Tamura, H. (2006). Scheduling multiple agents for picking products in a warehouse. Paper presented at the Robotics and Automation, 2006. ICRA 2006. Proceedings 2006 IEEE International Conference on.

Rushton, A., Croucher, P., \& Baker, P. (2014). The handbook of logistics and distribution management: Understanding the supply chain: Kogan Page Publishers.

Shukla, C., \& Frank Chen, F. (1996). The state of the art in intelligent real-time FMS control: a comprehensive survey. Journal of Intelligent Manufacturing, 7(6), 441-455. doi:10.1007/BF00122834

Staudt, F. H., Alpan, G., Di Mascolo, M., \& Rodriguez, C. M. T. (2015). Warehouse performance measurement: a literature review. International Journal of Production Research, 53(18), 5524-5544. doi:10.1080/00207543.2015.1030466

Tao, F., Cheng, J., Qi, Q., Zhang, M., Zhang, H., \& Sui, F. (2018). Digital twin-driven product design, manufacturing and service with big data. The International Journal of Advanced Manufacturing Technology, 94(9-12), 3563-3576.

Trab, S., Bajic, E., Zouinkhi, A., Thomas, A., Abdelkrim, M. N., Chekir, H., \& Ltaief, R. H. (2017). A communicating object's approach for smart logistics and safety issues in warehouses. Concurrent Engineering, 25(1), 53-67.

van den Berg, J. P., \& Zijm, W. H. (1999). Models for warehouse management: Classification and examples. International Journal of Production Economics, 59(1-3), 519-528.

Walker, M. (2018). Spotlight on the 7 key warehouse processes. MHD Supply Chain Solutions, $48(1), 20-22$. 
Weissbach, S., Radmanu, O., \& Grabowski, S. (2009). Handling Exceptional Situations in a Warehouse Management. In: Google Patents.

Widenius, M., Axmark, D., \& Arno, K. (2002). MySQL reference manual: documentation from the source: " O'Reilly Media, Inc.".

Zhang, L., Alharbe, N., \& Atkins, A. S. (2016). An IoT Application for Inventory Management with a Self-Adaptive Decision Model. Paper presented at the Internet of Things (iThings) and IEEE Green Computing and Communications (GreenCom) and IEEE Cyber, Physical and Social Computing (CPSCom) and IEEE Smart Data (SmartData), 2016 IEEE International Conference on.

Zhang, M., Batta, R., \& Nagi, R. (2009). Modeling of workflow congestion and optimization of flow routing in a manufacturing/warehouse facility. Management Science, 55(2), 267-280.

Copyright: (C) 2021 Binos, Bruno \& Adamopoulos. This is an open-access article distributed under the terms of the Creative Commons Attribution-NonCommercial 3.0 Australia License, which permits non-commercial use, distribution, and reproduction in any medium, provided the original author and AJIS are credited.

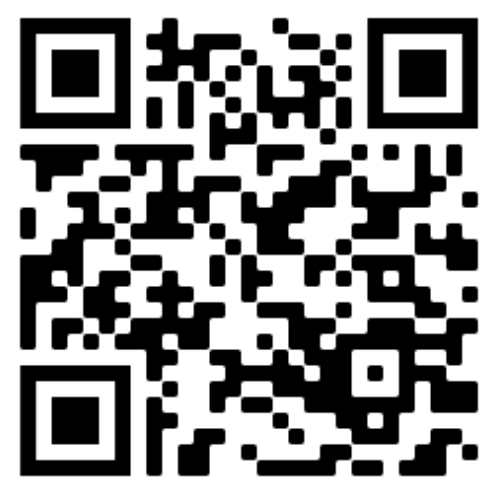

